International Journal of Pure and Applied Mathematics

Volume 110 No. 3 2016, 503-517

ISSN: 1311-8080 (printed version); ISSN: 1314-3395 (on-line version)

url: http://www.ijpam.eu

doi: 10.12732/ijpam.v110i3.10

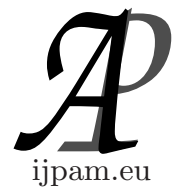

\title{
NUMERICAL STUDY OF 2D MHD CONVECTION AROUND PERIODICALLY PLACED CYLINDERS
}

\author{
Harijs Kalis ${ }^{1}$, Maksims Marinaki ${ }^{2}$ \\ ${ }^{1,2}$ Institute of Mathematics and Computer Science \\ University of Latvia \\ Raina bulvāris 29, Rīga LV-1459, LATVIA
}

\begin{abstract}
In this paper 2D stationary boundary value problem for the system of magnetohydrodynamic (MHD) equations along with the heat transfer equation is considered. The viscous electrically conducting incompressible liquid-electrolyte is to move between infinite cylinders placed periodically. Similarly 2D MHD channel flow with periodically placed obstacles on the channel walls is examined. We analyze the 2D MHD convection around the cylinders and obstacles subject to homogeneous external magnetics field. The cylinders, obstacles and walls of channel with constant temperature are heated.

The goal of such investigation is to obtain the distributions of stream function, temperature, velocity and the vortex formation in the cross-section plane of the cylinders and obstacles depending on the external magnetic field and on the direction of the gravitation. For the numerical treatment finite difference method is used.
\end{abstract}

AMS Subject Classification: 35Q35, 65N06, 65N22, 76W05, 76D17

Key Words: nonlinear PDE problem, finite-difference, MHD, heat transfer

\section{Introduction}

In many physical experiments and technological applications it is important to mix and heat an electroconductive liquid: liquid-metals, electrolyte, water, air. In the developed mathematical models vortex-type structures appearing in liquid flows, as well as in problems related to energy conversion in new technological devices.

Received: $\quad$ June 9, 2016

Revised: October 25, 2016

Published: November 5, 2016

$\S$ Correspondence author
(C) 2016 Academic Publications, Ltd. url: www.acadpubl.eu 
In the developed mathematical models, vortex-type structures appear in liquid flows, as well as in problems related to energy conversion in new technological devices. MHD convection flow of a viscous incompressible fluid around cylinder with combined effects of heat and mass transfer is an important problem prevalent in many engineering applications [1], [4], [5].

Heat exchanger systems are employed in numerous industries. Steam generation in boiler, air cooling within the coil of air conditioner and automotive radiators represent just some of the conventional applications of this mechanical system. Of particular importance in the design of heat exchanger is the pivotal understanding of heat transfer in flow across a bank of tubes. Tube banks, as used in many heat exchangers, can be systematically arranged in an in-line or staggered manner.

For mathematical model the flow around infinite periodically placed tube banks (cylinders with cross-section of the circle) can be considered [7]. In this paper the heat transfer significant influence on the fluid flow behaviour without the magnetic field and gravity is investigated. The problem is calculated using finite-volume CFD computer code ANSYS for the in-line and staggered arrangements of tube banks. The working fluid is taken to be water. Streamlines with two recirculation vortices at different Reynolds numbers $(R e=114-2735)$ are obtained. The lowest temperature is located within the mainstream bulk cold fluid while the highest temperature is located close to the circumferential wall.

We consider the viscous electrically conducting incompressible liquid-electrolyte. In [6] the flow and temperature of electrolyte in a conducting cylinder with alternating current is calculated.

The electrolyte is between infinite cylinders placed periodically in the $(x, y)$ plane. For the in-line arrangements of cylinders, the liquid has prescribed inlet ambient temperature $T_{0}$ much lower than the wall temperature $T_{w}$. By taking the advantage of special geometrical features the computational fluid domain allows the possible exploitation of symmetric and periodic boundary conditions in speeding up the computations and in turn enhancing the computational accuracy of the simplified geometries. Flow around finite arrangement of cylinders occurs in many applications (heat exchanges, fuel rod cooling etc). The formulation of single periodicity cell with periodic BCs is a reasonable approximation. Consider a closed flow circuit around cylinders in a periodic arrangement, then the temperature stationary field is going to be periodic in space.

The cross-sections of the cylinders are square. We consider 2D stationary boundary value problems for the system of magnetohydrodynamic (MHD) equation. We analyze the $2 \mathrm{D}$ free convection between these cylinders in homogeneous external magnetic field, depending on the direction of the gravitation. 
By taking the advantage of special geometrical features using the conditions of symmetry and periodicity, we can consider only two cylinders. Here, the cross - flow exchanger of cooler fluid re-wowing the heat from the warmer fluid within the tubes flowing perpendicular to the $2 \mathrm{D}$ plane.

This process of the magnetohydrodynamics (MHD) is considered with the so-called inductionless approximation. This would mean that the action of a moving liquid on the external magnetic field is insignificant [1].

The external magnetic field, Lorentz force, dimensionless stationary NavierStokes equations, numerical domain with two cylinders and the system of three equations for calculating the stream function, vorticity and temperature are formulated. The distribution of electromagnetic fields, forces, velocity and temperature around the cylinders has been calculated using the finite difference method, Seidel iterations and specific boundary conditions for the vorticity function.

\section{Mathematical Model}

The external homogeneous 2D magnetic field has two components of the induction:

$$
B_{x}=B_{0} \cos (\alpha), \quad B_{y}=B_{0} \sin (\alpha)
$$

where $\alpha$ is the angle between the Ox-axis and direction of the induction vector, $B_{0}$ is the magnitude of the magnetic field. The magnetic field creates the $F_{x}(t, x, y), F_{y}(t, x, y)$ components of the Lorentz force $\mathbf{F}$.

Considering the vector of Lorentz force $\mathbf{F}=\mathbf{J} \times \mathbf{B}, \mathbf{J}=\sigma(\mathbf{E}+\mathbf{V} \times \mathbf{B})$, we obtain

$$
\begin{gathered}
J_{z}=\sigma\left(E_{z}+B_{0}\left(V_{x} \sin (\alpha)-V_{y} \cos (\alpha)\right)\right), \\
F_{x}=-B_{y} J_{z}, \quad F_{y}=B_{x} J_{z}
\end{gathered}
$$

where $E_{z}=$ const, $J_{z}$ are the azimuthal components of the electric field vector $\mathbf{E}$ and the density vector of the electric current $\mathbf{J}, B_{x}, B_{y}$ are the components of the magnetic induction vector $\mathbf{B}, \sigma$ is the electric conductivity, $V_{x}, V_{y}$ are the components of velocity vector $\mathbf{V}$.

We analyze the free convection flow depending on two settings of the homogeneous magnetic field: the field parallel to Ox-axis $(\alpha=0)$ and the transverse field $\left(\alpha=\frac{\pi}{2}\right)$

The z-component $\frac{\partial F_{y}}{\partial x}-\frac{\partial F_{x}}{\partial y}$ of the vector curl $\mathbf{F}=\nabla \times \mathbf{F}$ affects the liquid motion, which can be described by Navier-Stokes equations in Boussinesq 
approximation and the heat transfer equation [2],[4]:

$$
\left\{\begin{array}{l}
\frac{\partial \mathbf{V}}{\partial t}+(\mathbf{V} \cdot \nabla) \mathbf{V}=-\frac{1}{\rho} \nabla p+\nu \Delta \mathbf{V}+\beta_{t} \mathbf{g}\left(T-T_{0}\right)+\frac{1}{\rho} \mathbf{J} \times \mathbf{B} \\
\nabla \cdot \mathbf{V}=0, \\
\frac{\partial T}{\partial t}+(\mathbf{V} \cdot \nabla) T=\frac{k}{\rho C_{p}} \Delta T+\frac{\left|J_{z}\right|^{2}}{\rho C_{p} \sigma}
\end{array}\right.
$$

where $\Delta$ is the Laplacian, $p, T, \sigma, \rho, \nu, k, C_{p}, \beta_{t}$ are static pressure, temperature, electrical conductivity, fluid density, kinematic viscosity, heat conductivity, specific heat, acceleration due to gravity, volumetric coefficient of thermal expansion, $T_{0}$ is the initial fluid temperature, $\mathbf{g}=g(\sin (\beta),-\cos (\beta), 0)$ is the vector of gravitation, $g$ is the gravitational acceleration, $\beta$ is the angle between the $O y$-axis and direction of the gravitation. The dynamic pressure is $p+p_{h}$, where $p_{h}$ is hydrostatical pressure.

The surface of the cylinders is subject to heat loss modelled by Newtonian cooling from the cylinders which is at a temperature $T_{w}>T_{0}$ with heat transfer coefficient $h\left[\frac{J}{s m^{2} K}\right]$ and there is resulting in the boundary conditions

$$
k \frac{\partial T}{\partial \vec{n}}=-h\left(T-T_{w}\right),
$$

where $n$ is the external normal to the surface of the cylinders.

The equations (1) were made dimensionless by using characteristic values $L_{0}$ (side length of a square), $U_{0}$ (velocity), $B_{0}$ (magnetic field), $P_{0}=U_{0}^{2} \rho$ (pressure), $L_{0} / V_{0}$ (time), $U_{0} B_{0}$ (density vector of the electric current). The dimensionless temperature is equal to $\frac{T-T_{0}}{T_{w}-T_{0}} \in[0,1]$.

The dimensionless boundary conditions for temperature on $r=1$ is

$$
\frac{\partial T}{\partial \vec{n}}+B i(T-1)=0
$$

where $B i=\frac{h L_{0}}{\lambda}$ is the Biot number.

Using the vorticity function $\zeta=\frac{\partial V_{y}}{\partial x}-\frac{\partial V_{x}}{\partial y}$, one obtains

$$
\left\{\begin{array}{l}
\frac{\partial V_{x}}{\partial t}-\zeta V_{y}=-\frac{\partial \bar{p}}{\partial x}-\frac{1}{R e} \frac{\partial \zeta}{\partial y}-\frac{G r}{R e^{2}} T \sin (\beta)-S \sin (\alpha) j_{z} \\
\frac{\partial V_{y}}{\partial t}+\zeta V_{x}=-\frac{\partial \bar{p}}{\partial y}+\frac{1}{R e} \frac{\partial \zeta}{\partial x}+\frac{G r}{R e^{2}} T \cos (\beta)+S \cos (\alpha) j_{z} \\
\frac{\partial V_{x}}{\partial x}+\frac{\partial V_{y}}{\partial y}=0 \\
\frac{\partial T}{\partial t}+V_{x} \frac{\partial T}{\partial x}+V_{y} \frac{\partial T}{\partial y}=\frac{1}{P e} \Delta T+\frac{K_{T}}{P e} j_{z}^{2}
\end{array}\right.
$$


where $j_{z}=e_{z}+V_{x} \sin (\alpha)-V_{y} \cos (\alpha), e_{z}$ is the dimensionless form of azimuthal component for electric current density and electric field, $\bar{p}=p+0.5 \mathbf{V}^{2}$,

$$
\begin{gathered}
R e=\frac{U_{0} L_{0}}{\nu}, \quad S=\frac{\sigma B_{0}^{2} L_{0}}{\rho U_{0}}, \quad G r=\frac{\beta_{t} g\left(T_{w}-T_{0}\right) L_{0}^{3}}{\nu^{2}}, \\
H a=\sqrt{\operatorname{Re} S}=L_{0} B_{0} \sqrt{\frac{\sigma}{\rho \nu}}
\end{gathered}
$$

are Reynolds, Stuart, Grashof and Hartman numbers, $\operatorname{Pe}=\operatorname{Pr} \operatorname{Re}, \operatorname{Pr}=\frac{\nu \rho C_{p}}{k}$, $K_{T}=\frac{\sigma B_{0}^{2} L_{0}^{2} U_{0}^{2}}{k\left(T_{w}-T_{0}\right)}$ are Prandtl number and heat source parameter.

The hydrodynamic stream function $\psi$ can be determined by relations

$$
V_{x}=\frac{\partial \psi}{\partial y}, \quad V_{y}=-\frac{\partial \psi}{\partial x}
$$

Eliminating the pressure from Eq. (4), one obtains

$$
\left\{\begin{array}{l}
\frac{\partial \zeta}{\partial t}-J(\psi, \zeta)=\frac{1}{R e} \Delta \zeta+\frac{G r}{R e^{2}}\left(\frac{\partial T}{\partial x} \cos (\beta)+\frac{\partial T}{\partial y} \sin (\beta)\right)+S f \\
\zeta=-\Delta \psi \\
\frac{\partial T}{\partial t}-J(\psi, T)=\frac{1}{P e} \Delta T+\frac{K_{T}}{P e} j_{z}^{2}
\end{array}\right.
$$

where

$$
f=\sin (2 \alpha) \frac{\partial^{2} \psi}{\partial x \partial y}+\cos ^{2}(\alpha) \frac{\partial^{2} \psi}{\partial x^{2}}+\sin ^{2}(\alpha) \frac{\partial^{2} \psi}{\partial y^{2}}
$$

is the z-component of the vector $\operatorname{curl} \mathbf{F}$,

$$
J(\psi, v)=\frac{\partial \psi}{\partial x} \frac{\partial v}{\partial y}-\frac{\partial \psi}{\partial y} \frac{\partial v}{\partial x}
$$

is the Jacobian of the functions $\psi, v, v=\zeta ; T$,

$$
j_{z}=e_{z}+\frac{\partial \psi}{\partial l_{B}}
$$

is the derivative in the direction

$$
l_{B}=(\cos (\alpha), \cos (\pi / 2-\alpha), 0)
$$

Using the boundary conditions (BCs) of symmetry and periodicity, we can consider only the domain containing quarters of two cylinders, see [9]. 
For the MHD convection using in-line arrangement cylinders and periodic flow (PCF) we consider the domain (see [2]) $\Omega=\Omega_{1} \bigcup \Omega_{2}$ (see Figures $1,2)$, where

$$
\begin{gathered}
\Omega_{1}=\left\{(x, y): l_{1} \leq x \leq l_{2}, 0 \leq y \leq L_{1}\right\}, \\
\Omega_{2}=\left\{(x, y): 0 \leq x \leq l, L_{1} \leq y \leq L\right\}, 0<l_{1}<l_{2}<l, 0<L_{1}<L .
\end{gathered}
$$

Here $C_{1}=\left\{(x, y): 0<x<l_{1}, 0<y<L_{1}\right\}$ and $C_{2}=\left\{(x, y): l_{2}<x<l, 0<\right.$ $\left.y<L_{1}\right\}$ are the quarters of cylinders, $L^{1}=\{(x, L): 0 \leq x \leq l\}, L^{2}=\{(x, 0)$ : $\left.l_{1} \leq x \leq l_{2}\right\}$ are the plane of symmetry with BCs $V_{y}=0, \frac{\partial T}{\partial y}=\zeta=0, \psi=\psi_{0}$ on $L^{1}, \psi=0$ on $L^{2}$,

$$
\begin{gathered}
W^{1}=\left\{\left(x, L_{1}\right): 0<x \leq l_{1}\right\}, \quad W^{2}=\left\{\left(x, L_{1}\right): l_{2} \leq x<l\right\}, \\
W^{3}=\left\{\left(l_{1}, y\right): 0<y \leq L_{1}\right\} \text { and } W^{4}=\left\{\left(l_{2}, y\right): 0<y \leq L_{1}\right\}
\end{gathered}
$$

are the walls of the cylinders with the non-slip BCs $V_{x}=V_{y}=\psi=0, I_{n}=$ $\left\{(0, y): L_{1}<y \leq L\right\}$ is the inlet and $O_{t}=\left\{(l, y): L_{1}<y \leq L\right\}$ is the outlet with the periodical BCs for $\psi, \zeta, T, U_{x}, U_{y}$.

In the case of free MHD convection, $\psi_{0}=0$.

For the additional periodic channel flow with symmetry (CF),

$$
L^{2}=W^{5}=\left\{(x, 0): l_{1} \leq x \leq l_{2}\right\}
$$

is the wall of the half-channel $\Omega$ with non-slip BCs, $V_{x}=V_{y}=\psi=0$.

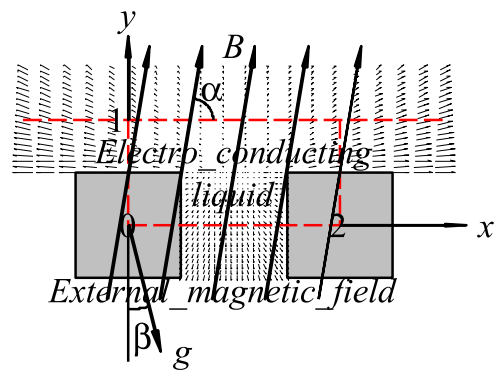

Figure 1: Domain for in-line placed cylinders $\left(2\right.$ cylinders, $L_{1}=$ $0.5, L=1, l_{1}=0.5, l_{2}=1.5, l=2$ )

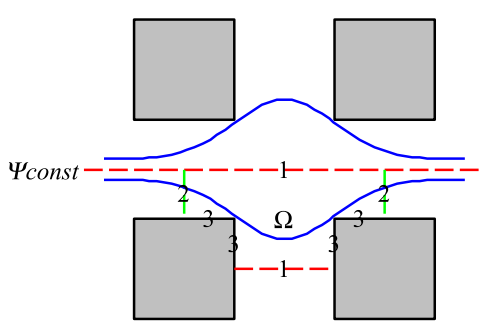

Figure 2: Domain for in-line placed cylinders (4 cylinders, BCs: 1symetry,2- periodic, 3 -walls) 
The cylinders are electrically non-conducting and

$$
\int_{-\infty}^{\infty} \int_{-\infty}^{\infty} j_{z} d x d y=0 .
$$

From

$$
\int_{\Omega}\left(\frac{\partial \psi}{\partial l_{B}}+e_{z}\right) d x d y=0
$$

it follows that

$$
e_{z}=-\frac{1}{m e s \Omega} \int_{\partial \Omega} \psi \cos \left(n, l_{B}\right) d s
$$

or

$$
e_{z}=-\frac{\psi_{0} l \sin (\alpha)}{L_{1}\left(l_{2}-l_{1}\right)+l\left(L-L_{1}\right)} .
$$

On the walls we use the following BCs [3]:

$$
\zeta^{m}=\gamma \frac{\partial \psi}{\partial n}+\zeta^{m-1}, m=1,2, \cdots,
$$

where $m$ is the number of iterations with $\zeta^{0}=0, \gamma>0$ is the parameter, $n$ is the outer normal to the walls.

We consider following parameters for electrolyte [8]: $T_{w}-T_{0}=70[K]$, $\left(T_{0}=300[K], T_{w}=370[K]\right), \rho=1100\left[\frac{\mathrm{kg}}{\mathrm{m}^{3}}\right], C_{p}=4000\left[\frac{\mathrm{J}}{\mathrm{kgK}}\right], \nu=1 . e-6\left[\frac{\mathrm{m}^{2}}{\mathrm{~s}}\right]$, $k=1.1\left[\frac{W}{m K}\right], \sigma=100\left[\frac{1}{\Omega m}\right], h=0.44\left[\frac{J}{s m^{2} K}\right], \beta_{t}=2 . e-4\left[\frac{1}{K}\right]$.

The characteristic length scale is $L_{0}=0.063[\mathrm{~m}]$ (the diameter of cylinder).

For flow through periodically placed cylinders arranged in-line, with $G r=0$, similarly to [2], in a space between cylinders the vortexes are created.

For free convection flow we assume that $R e=1$ and we have the magnitude for the velocity $U_{0}=\frac{\nu}{L_{0}}=0.16 e-4\left[\frac{\mathrm{m}}{\mathrm{s}}\right]$.

Using Stuart number we have $S=400, B_{0}^{2}=0 ; 100 ; 1000$ for $B_{0}=$ $0 ; 0.5 ; 1.58$ and $H a=0 ; 10 ; 31.6$.

In order to obtain the dimensional values, we need to multiply the dimensionless values by the following scalar factors:

1) the values of velocity with $U_{0}=0.16 e-4$,

2) the vorticity with $U_{0} / L_{0}=0.25 e-3$,

3) the stream function with $U_{0} L_{0}=1 . e-6$,

4)the pressure with $\rho U_{0}^{2}=0.26 e-5$.

The dimensional temperature is $T_{0}+\left(T_{w}-T_{0}\right) T=300+70 T[K]$. The heat source parameter $K_{T} \approx 1 . e-10$ is negligible. 


\section{Numerical Algorithm for Solution of the Problem}

We consider an uniform grid $((N+1) \times M)$ :

1) $\Omega_{1}^{h}=\left\{\left(x_{i}, y_{j}\right), x_{i}=(i-1) h, y_{j}=(j-1) h\right\}, i=\overline{N_{1}, N_{2}}, j=\overline{1, M_{1}}$, $\left(N_{1}-1\right) h=l_{1},\left(M_{1}-1\right) h=L_{1}$;

2) $\Omega_{2}^{h}=\left\{\left(x_{i}, y_{j}\right), x_{i}=(i-1) h, y_{j}=(j-1) h\right\}, i=\overline{1, N+1}, j=$ $\overline{M_{1}, M},(N-1) h=l_{1},(M-1) h=L$;

where $h=\frac{l_{1}}{N_{1}-1}=\frac{l_{2}}{N_{2}-1}=\frac{l}{N-1}=\frac{L}{M-1}=\frac{L_{1}}{M_{1}-1}$. Subscripts $(i, j)$ refer to $x, y$ indices with the mesh spacing $h$.

Equations (5) in the uniform grid $\left(x_{i}, y_{j}\right)$ are replaced by difference equations of second order approximation in a 5-point stencil and the numerical calculations are made using Seidel iterations with under-relaxation for vorticity and temperature (see Appendix, where $\Psi_{i, j} \approx \psi\left(x_{i}, y_{j}\right), \zeta_{i, j} \approx \zeta\left(x_{i}, y_{j}\right), T_{i, j} \approx$ $\left.T\left(x_{i}, y_{j}\right)\right)$. The difference of pressure $P=p(l, L)-p(l, 0), p(l, 0)=0$ and integral heat quantity $Q_{T}=\int_{\Omega} T(x, y) d x d y$ are calculated (the maximal value is $Q_{T}=1.500$ for maximal dimensionless temperature $T \equiv 1$ ).

\section{Some Numerical Results}

Numerical results are obtained for $l_{1}=0.5, l_{2}=1.5, l=2, L=1, L_{1}=0.5$, $\beta=0 ; \pm \frac{\pi}{2}, \alpha=0 ; \frac{\pi}{2}, \gamma=1$.

The calculations and their graphical visualization were made by means of the MATLAB software for 2 different grids:

$$
\begin{aligned}
& \text { 1) } h=0.0125, N_{1}=41, N_{2}=121, N=161, M_{1}=41, M=81, \\
& 2) h=0.00625, N_{1}=81, N_{2}=241, N=321, M_{1}=81, M=161 .
\end{aligned}
$$

For default calculation we use the grid Nr. 1 with $N=161, M=81$.

The iterative process with maximal errors $\leq 10^{-6}$ for $\Psi$ and $\leq 10^{-3}$ for $\zeta$ and temperature depends on the parameters (the number of iterations $I t=$ $10000-100000)$. In the corresponding figures we can see the levels of stream function $\Psi=$ const, temperature $T=$ const, velocity $V=$ const and vorticity $\zeta=$ const.

For the free convection flow for PCF and $\mathrm{CF}$ we consider $\beta=0 ; \pm \frac{\pi}{2}, S=$ $0 ; 100 ; 1000, \alpha=0 ; \frac{\pi}{2}, G r=1 . e+5, \operatorname{Pr}=4$.

In the Table 1 the maximal (Mvx, Mvy) and minimal (mvx, mvy) values of velocity $\left(V_{x}, V_{y}\right)$, stream function $(m \Psi)$, vorticity $(M|\zeta|)$, integral heat quantity $\left(Q_{T}\right)$ and difference of pressure $(P)$ for $G r=1 . e+5$ are represented. 
Table 1: The values of $\beta, S, \alpha, M v x, m v x, M v y, m v y, m \Psi, M|\zeta|, Q_{T}, P$ for $G r=1 . e+5$

\begin{tabular}{|c|c|c|c|c|c|c|c|c|c|c|}
\hline$\beta$ & $S$ & $\alpha$ & $M v x$ & $M v y$ & $m v x$ & mvy & $m \Psi$ & $M|\zeta|$ & $Q_{T}$ & $P$ \\
\hline$C F,-\frac{\pi}{2}$ & 0 & - & 5.12 & 4.84 & -5.68 & -4.53 & 1.480 & 246 & 1.469 & $1.95 e+5$ \\
\hline$C F, \frac{\pi^{2}}{2}$ & 0 & - & 5.68 & 4.84 & -5.12 & -4.53 & $-1 ; 480$ & 246 & 1.469 & $-1.95 e+5$ \\
\hline$C F,-\frac{\pi}{2}$ & 100 & 0 & 5.03 & 4.28 & -6.19 & -3.96 & 1.255 & 299 & 1.465 & $1.94 e+5$ \\
\hline$C F, \frac{\pi^{2}}{2}$ & 100 & 0 & 6.19 & 4.28 & -5.03 & -3.96 & -1.255 & 299 & 1.465 & $-1.94 e+5$ \\
\hline$C F,-\frac{\pi}{2}$ & 1000 & 0 & 6.78 & 2.68 & -8.01 & -2.45 & $1.703^{1}$ & 432 & 1.458 & $1.93 e+5$ \\
\hline$C F, \frac{\pi}{2}$ & 1000 & 0 & 8.01 & 3.68 & -6.78 & -2.45 & $-1.703^{1}$ & 432 & 1.458 & $-1.93 e+5$ \\
\hline$C F,-\frac{\pi}{2}$ & 100 & $\frac{\pi}{2}$ & 3.22 & 2.93 & -2.68 & -2.87 & .840 & 166 & 1.468 & $1.96 e+5$ \\
\hline$C F, \frac{\pi^{2}}{2}$ & 100 & $\frac{\pi}{2}$ & 2.68 & 2.93 & -3.22 & -2.87 & -.840 & 166 & 1.468 & $-1.96 e+5$ \\
\hline$C F,-\frac{\pi}{2}$ & 1000 & $\frac{\pi}{2}$ & 1.00 & 1.21 & -.72 & -1.33 & 0.257 & 92.5 & 1.465 & $1.98 e+5$ \\
\hline$C F, \frac{\pi^{2}}{2}$ & 1000 & $\frac{\pi}{2}$ & .72 & 1.21 & -1.00 & -1.33 & -0.257 & 92.5 & 1.465 & $-1.98 e+5$ \\
\hline$C F, 0$ & 0 & & 2.18 & 2.28 & -2.18 & -2.29 & \pm 0.424 & 135.3 & 1.482 & 0 \\
\hline$C F, 0$ & 100 & $\frac{\pi}{2}$ & 1.34 & 1.99 & -1.34 & -2.01 & \pm 0. & 113 & 1.481 & 0 \\
\hline$C F, 0$ & 1000 & $\frac{\pi}{2}$ & .46 & .84 & -.46 & -.83 & \pm 0.130 & 57.6 & 1.476 & 0 \\
\hline$C F, 0$ & 1000 & 0 & .77 & .34 & -.77 & -.34 & \pm 0.105 & 47.2 & 1.473 & 0 \\
\hline$P C F,-\frac{\pi}{2}$ & 0 & - & 12.14 & 8.45 & -10.12 & -8.59 & 2.694 & 521 & 1.419 & $1.89 e+5$ \\
\hline$P C F, \frac{\pi^{2}}{2}$ & 0 & - & 10.12 & 8.45 & -12.14 & -8.59 & -2.694 & 521 & 1.419 & $-1.89 e+5$ \\
\hline$P C F,-\frac{\pi}{2}$ & 100 & 0 & 10.08 & 6.93 & -11.62 & -7.28 & 2.208 & 633 & 1.414 & $1.87 e+5$ \\
\hline$P C F, \frac{\pi^{2}}{2}$ & 100 & 0 & 11.62 & 6.93 & -10.08 & -7.28 & -2.208 & 633 & 1.414 & $-1.87 e+5$ \\
\hline$P C F,-\frac{\pi}{2}$ & 1000 & 0 & 10.98 & 3.71 & -12.47 & -3.76 & $1.893^{1}$ & 706 & 1.412 & $1.86 e+5$ \\
\hline$P C F, \frac{\pi}{2}$ & 1000 & 0 & 12.47 & 3.71 & -10.98 & -3.76 & $-1.893^{1}$ & 706 & 1.412 & $-1.86 e+5$ \\
\hline$P C F,-\frac{\pi}{2}$ & 100 & $\frac{\pi}{2}$ & 6.17 & 4.12 & -5.02 & -5.12 & 1.392 & 315 & 1.415 & $1.88 e+5$ \\
\hline$P C F, \frac{\pi^{2}}{2}$ & 100 & $\frac{\pi}{2}$ & 5.02 & 4.12 & -6.17 & -5.12 & -1.392 & 315 & 1.415 & $-1.88 e+5$ \\
\hline$P C F,-\frac{\pi}{2}$ & 1000 & $\frac{\pi}{2}$ & 1.26 & 1.39 & -1.06 & -2.14 & 0.363 & 163 & 1.409 & $1.90 e+5$ \\
\hline$P C F, \frac{\pi^{2}}{2}$ & 1000 & $\frac{\pi}{2}$ & 1.06 & 1.39 & -1.26 & -2.14 & -0.363 & 163 & 1.409 & $-1.90 e+5$ \\
\hline$P C F, 0$ & 0 & 2 & 4.27 & 3.63 & -4.27 & -3.62 & \pm 0.679 & 242.2 & 1.441 & 0 \\
\hline$P C F, 0$ & 100 & $\frac{\pi}{2}$ & 2.78 & 3.48 & -2.78 & & \pm 0.559 & 234 & 1.440 & 0 \\
\hline$P C F, 0$ & 1000 & $\frac{\pi}{2}$ & 1.38 & 2.31 & -1.38 & -1.74 & \pm 0.327 & 172 & 1.431 & 0 \\
\hline$P C F, 0$ & 1000 & 0 & 2.03 & .91 & -2.03 & -.92 & \pm 0.288 & 137 & 1.430 & 0 \\
\hline
\end{tabular}

In the Figures 3-14 we can see the results of calculations for the free convection $\mathrm{CF}$ and PCF flow for $R e=1, \operatorname{Pr}=4, G r=1 . e+5, S=0 ; 100 ; 1000, \alpha=$ $\frac{\pi}{2} ; 0, \beta=0 ; \pm \frac{\pi}{2}$.

The gravitation in $\mathrm{x}$-direction determine the direction of velocity and orientation of vortexes in the flow. For $\beta=\frac{\pi}{2}$ the central flow is moving in x-direction and the vortex between cylinder rotated clockwise, but for $\beta=-\frac{\pi}{2}$ the central flow is moving in opposite $\mathrm{x}$-direction and the vortex between cylinder rotated counter-clockwise (see Figures 9,10). For temperature we can consider similar behaviour (see Figures 11,12). The flow for $\beta=\frac{\pi}{2}$ is obtained from the flow for $\beta=-\frac{\pi}{2}$ by replacing the velocity $V_{x}$ with $-V_{x}$ and the extreme values $M v x$ with $-m v x$, $m v x$ with $-M v x$ and $P$ with $-P$ (values $Q_{T}, M|\zeta|, M v y, m v y$ remain unchanged). The integral heat quantity (from $Q_{T}=1.377$ to $Q_{T}=1.362$ ) decreases in the magnetic field:

1) for $\beta= \pm \frac{\pi}{2} \mathrm{CF}-Q_{T}=1.458(S=1000) \div Q_{T}=1.469(S=0)$, PCF- 
$Q_{T}=1.409(S=1000) \div Q_{T}=1.419(S=0)$,

2) for $\beta=0, P=0 \mathrm{CF}-Q_{T}=1.473(S=1000) \div Q_{T}=1.482(S=0)$, PCF$Q_{T}=1.430(S=1000) \div Q_{T}=1.441(S=0)$.

The temperature is greater for $\mathrm{CF}$ convection (see Figures 3-6). For $\beta=0$ we have two symmetrical vortexes (see Figures 7, 8, 13). Figure 14 for $C F, \alpha=$ $0, S=1000, \beta=-\frac{\pi}{2}$ represents two opposite flows without vortices. Similarly the flow for PCF is obtained (see Table 1 the values of $m \Psi$ with index 1).

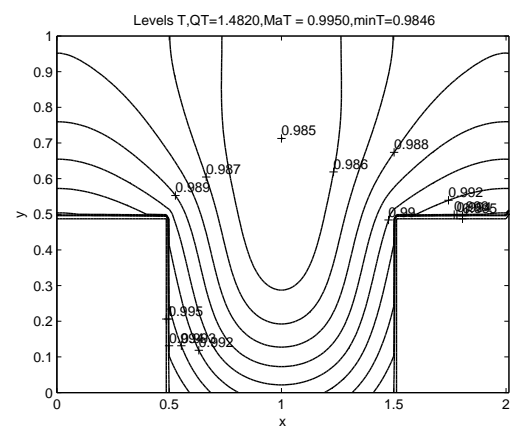

Figure 3: Levels of temperature for free convection $\mathrm{CF}$ flow for $S=$ $0, G r=1 . e+5, \beta=0$

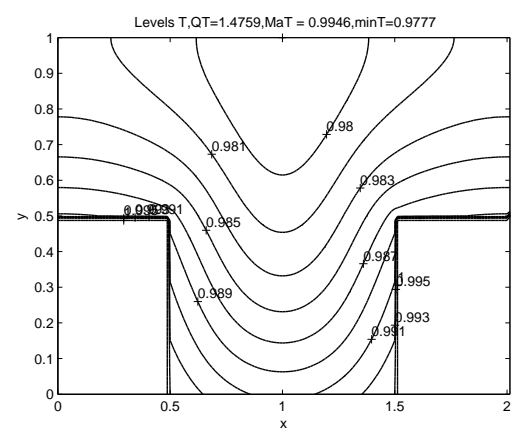

Figure 5: Levels of temperature for free convection $\mathrm{CF}$ flow for $\alpha=$ $\frac{\pi}{2}, S=1000, G r=1 . e+5, \beta=0$

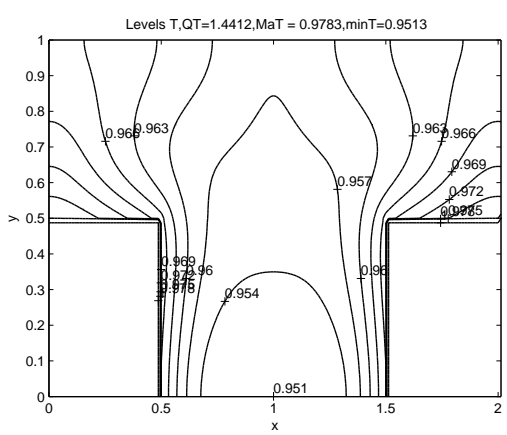

Figure 4: Levels of temperature for free convection PCF flow for $S=$ $0, G r=1 . e+5, \beta=0$

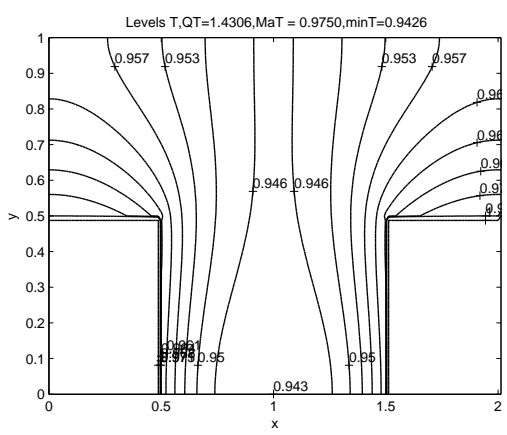

Figure 6: Levels of temperature for free convection PCF flow for $\alpha=$ $\frac{\pi}{2}, S=1000, G r=1 . e+5, \beta=0$ 


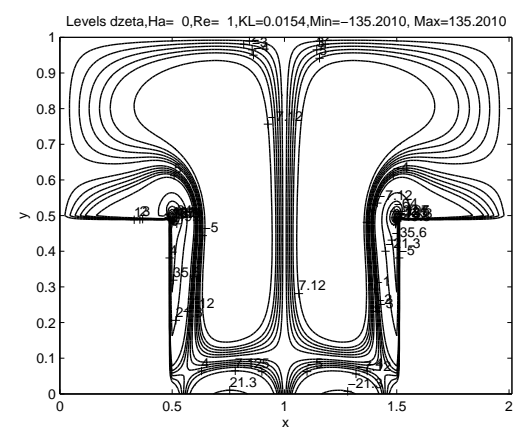

Figure 7: Levels of vorticity for free convection $\mathrm{CF}$ flow for $S=0, G r=$ 1.e $+5, \beta=0$

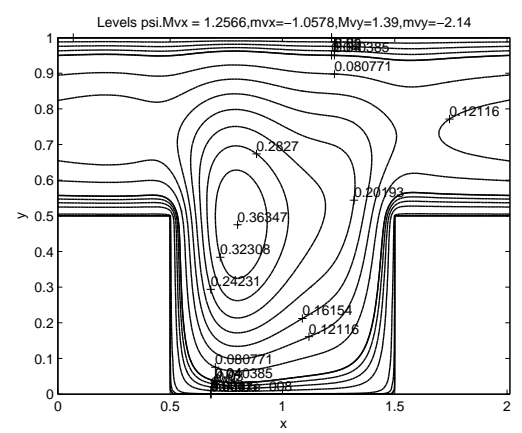

Figure 9: Levels of stream function for free convection PCF flow for $\alpha=$ $\frac{\pi}{2}, S=1000, G r=1 . e+5, \beta=-\frac{\pi}{2}$

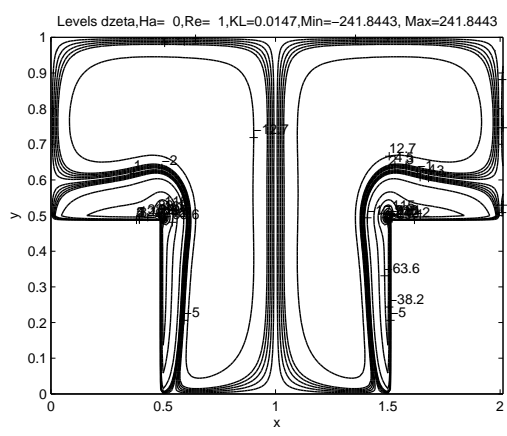

Figure 8: Levels of vorticity for free convection PCF flow for $S=$ $0, G r=1 . e+5, \beta=0$

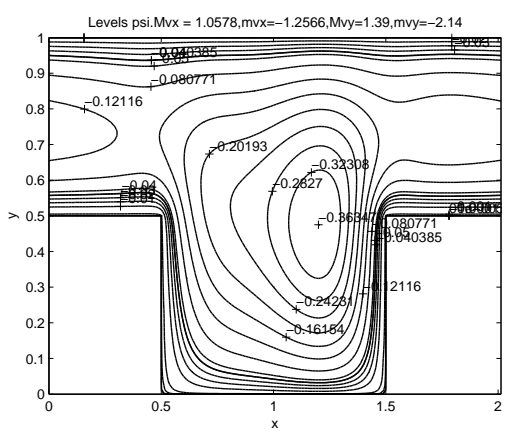

Figure 10: Levels of stream function for free convection PCF flow for $\alpha=$ $\frac{\pi}{2}, S=1000, G r=1 . e+5, \beta=\frac{\pi}{2}$ 


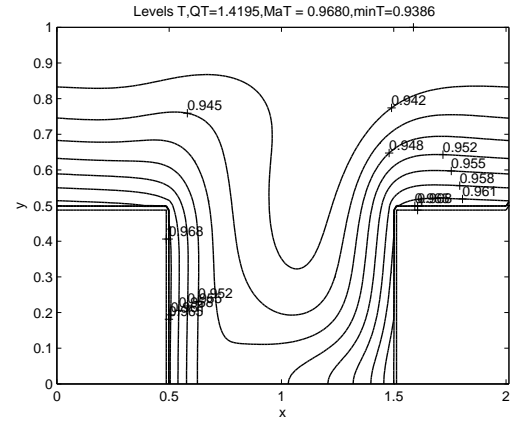

Figure 11: Levels of temperature for free convection PCF flow for $S=$ $0, G r=1 . e+5, \beta=\frac{\pi}{2}$

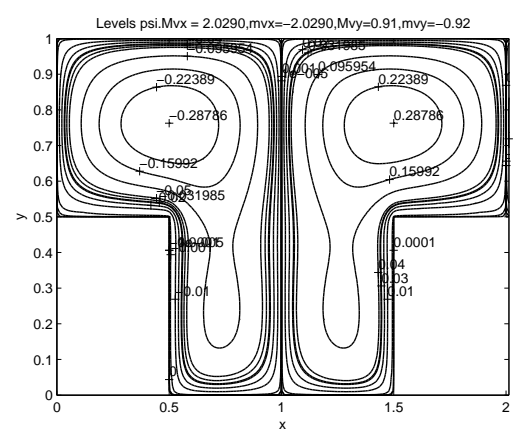

Figure 13: Levels of stream function for free convection PCF flow for $\alpha=$ $0, S=1000, G r=1 . e+5, \beta=0$

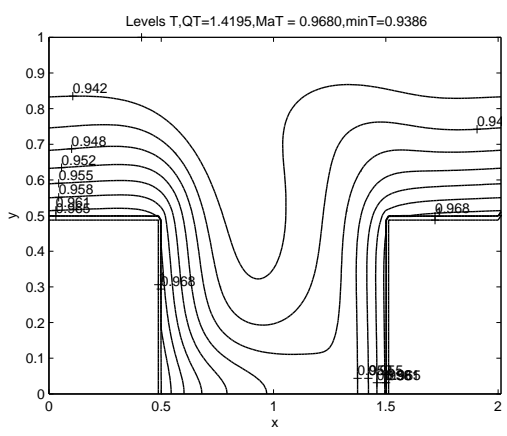

Figure 12: Levels of temperature for free convection PCF flow for $S=$ $0, G r=1 . e+5, \beta=-\frac{\pi}{2}$

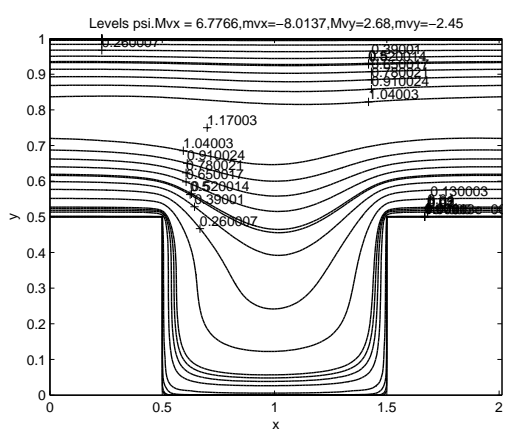

Figure 14: Levels of stream function for free convection $\mathrm{CF}$ flow for $\alpha=$ $0 S=1000, G r=1 . e+5, \beta=-\frac{\pi}{2}$ 


\section{Conclusions}

- The 2D MHD free convection flow and temperature fields have been calculated in case of periodically placed cylinders $(\mathrm{PCF})$ and obstacles in the channel (CF) for Grashof number's values $G r=10^{5}$, for Stuart number's values $S=0 ; 100 ; 1000$, for Prandtl number's value $\operatorname{Pr}=4$, for different directions of uniform magnetic field ( $\alpha=0$ - parallel to flow, $\alpha=\frac{\pi}{2}$ transverse) and for different orientation of the gravitational force $(\beta=0$ - transverse, $\beta=\frac{\pi}{2} ;-\frac{\pi}{2}$ - parallel to the flow with direct and opposite direction).

- In the transverse magnetic field $\left(\alpha=\frac{\pi}{2}\right)$ and gravitation $(\beta=0)$ we have symmetrical flow and temperature corresponding to y-axis (see Figures $3-8)$.

- If $\alpha= \pm \frac{\pi}{2}$, then vortexes and integral heat quantity decrease in the magnetic field; for $\alpha=0$ the vorticity increases but the integral heat quantity decreases in the magnetic field.

- Depending on orientation of gravity we have different behaviour of flow: The gravitation in $\mathrm{x}$-direction determine the direction of velocity, temperature and orientation of vortexes in the flow (see Figures 9-12), for $\beta=\frac{\pi}{2}$ the central flow is moving in $\mathrm{x}$-direction and the vortex between cylinder rotated clockwise, but for $\beta=-\frac{\pi}{2}$ the central flow is moving in opposite direction and the vortex between cylinder rotated counter-clockwise, similar behaviour can be observed for temperature.

- The integral heat quantity for $\mathrm{CF}$ convection is greater compared with PCF convection (see Tab. 1).

- In the parallel magnetic field vortices diminish and for $\alpha=0, S=1000, \beta=$ $\pm \frac{\pi}{2}$ we obtain the vortex-free flow with two opposite flows in x-direction (see Figure 14, Tab. 1). 


\section{Appendix}

The difference equations of second order approximation in 5-point stencil are in following form:

$$
\left\{\begin{array}{l}
4 \Psi_{i, j}=\bar{S} \Psi_{i, j}+h^{2} \zeta_{i, j} \\
\frac{R e}{4} J_{i, j}(\Psi, \zeta)-4 \zeta_{i, j}+\bar{S} \zeta_{i, j} \\
\quad=H a^{2}\left((\sin (\alpha))^{2} d_{y}^{2} \Psi_{i, j}+(\cos (\alpha))^{2} d_{x}^{2} \Psi_{i, j}-0.25 \sin (2 \alpha) d_{x, y}^{2} \Psi_{i, j}\right) \\
\quad-\frac{G r h}{R e}\left(\cos (\beta) d_{x} T_{i, j}+\sin (\beta) d_{y} T_{i, j}\right) \\
\frac{P e}{4} J_{i, j}(\Psi, T)-4 T_{i, j}+\bar{S} T_{i, j}=-K_{T} h^{2}\left(e_{z}+h^{-1}\left(\cos (\alpha) d_{x} \Psi_{i, j}\right.\right. \\
\left.\quad+\sin (\alpha) d_{y} \Psi_{i, j}\right)^{2}
\end{array}\right.
$$

where $\bar{S} q_{i, j}=q_{i, j-1}+q_{i, j+1}+q_{i-1, j}+q_{i+1, j}, q=\Psi ; T ; \zeta$,

$d_{x}^{2} \Psi_{i, j}=2 \Psi_{i, j}-\Psi_{i+1, j}-\Psi_{i-1, j}, d_{y}^{2} \Psi_{i, j}=2 \Psi_{i, j}-\Psi_{i, j+1}-\Psi_{i, j-1}$,

$d_{x y}^{2} \Psi_{i, j}=\Psi_{i+1, j+1}+\Psi_{i-1, j-1}-\Psi_{i-1, j+1}-\Psi_{i+1, j-1}$,

$d_{x} q_{i, j}=0.5\left(q_{i+1, j}-q_{i-1, j}\right), d_{y} q_{i, j}=0.5\left(q_{i, j+1}-q_{i, j-1}\right), q=\Psi ; T$,

$J_{i, j}(\Psi, q)=\left(\Psi_{i+1, j}-\Psi_{i-1, j}\right)\left(q_{i, j+1}-q_{i, j-1}\right)-\left(q_{i+1, j}-q_{i-1, j}\right)\left(\Psi_{i, j+1}-\Psi_{i, j-1}\right)$, $q=\zeta ; T$.

The numerical calculation of (6) is evaluated using Seidel iterations with underrelaxation for $\zeta, T$ functions:

$$
\begin{gathered}
\zeta_{i, j}^{m}=\omega_{1} \zeta_{i, j}^{z}+\left(1-\omega_{1}\right) \zeta_{i, j}^{m-1} \\
T_{i, j}^{m}=\omega_{2} T_{i, j}^{z}+\left(1-\omega_{2}\right) T_{i, j}^{m-1}, \quad m=1,2, \cdots,
\end{gathered}
$$

where $\zeta_{i, j}^{z}, T_{i, j}^{z}$ are the grid function value in central mesh points, obtained for m-th iteration, $\omega_{1}, \omega_{2} \in(0.1,0.5)$ are the under relaxation coefficients.

The discrete BCs [3] with $O\left(h^{2}\right)$ on the walls $w$ are computed in following form:

$$
\zeta_{w}^{m}=\frac{\gamma}{2 h}\left(-4 \Psi_{w-1}^{m}+\Psi_{w-2}^{m}+3 \Psi_{w}\right)+\zeta_{w}^{m-1},
$$

where $\Psi_{w-1}^{m}, \Psi_{w-2}^{m}$ are the value of $\Psi_{i, j}$ for one and two steps $h$ distant from the wall in the inner normal direction. On the corner of wall the value of $\zeta$ is equal to average value of the two nearest $\zeta$ values of the wall.

We determine the gradient of the pressure $p^{*}=p+0.5 \mathbf{V}^{2}$ used in the equations (4). On the symmetry plane $y=L$ from the first equation (1) $\left(V_{y}=\zeta=0, \Psi=0\right)$ it follows that:

$$
{\frac{\partial p^{*}}{\partial x}}_{i, M}
$$




$$
=-\frac{1}{2 R e h}\left(\zeta_{i, M-2}-4 \zeta_{i, M-1}\right)-\frac{G r}{R e^{2}} T_{i, M} \sin (\beta)-S \sin (\alpha)\left(V x_{i, M} \sin (\alpha)+e_{z}\right) .
$$

The pressure $p=p^{*}-0.5 \mathbf{V}^{2}$ is calculated using trapezoidal quadrature formula in the direction from the point $(0, L)$ to point $(l, L)$, assumed that $p(0, L)=0$.

\section{Acknowledgements}

Authors gratefully acknowledge the partial support from the grant Nr. 623/2014 of the Latvian Council of Science.

\section{References}

[1] Ju.M. Geljfgad, O.A. Lielausis, E.V. Cherbinin, Liquid Metal in the Action of Electromagnetic Forces, Zinatne, Riga, 1976.

[2] H. Kalis, M. Marinaki, A. Gedroics, Mathematical modelling of 2D MHD flow around infinite cylinders with square section placed periodically, Magnetohydrodynamics - MHD, 48, No. 3 (2012), 527-542.

[3] A.A. Dorodnycin, N.A.Meller, On some methods for solving Navier-Stokes equations, In: Abstr. of 3-th Congress of Theoretical and Applied Mechanics, Moscow, 1968.

[4] A.B. Vatatchyn, G.A. Ljubimov, S.A. Regirer, Magnetohydrodinamic Flows in a Channel, Nauka, Moscow, 1970.

[5] H.E. Kalis, A.B. Cinober, On deformation of hydrodynamical perturbation in uniform magnetic field, Magnetohydrodynamics, 2 (1972), 25-28.

[6] A. Buikis, H. Kalis, Flow and temperature calculations of electrolyte for a finite cylinder in the alternating field of finite number circular wires, Magnetohydrodynamics-MHD, 40, No. 1 (2004), 77-90.

[7] J. Tu, G.H. Yeoh, C. Liu, Computational Fluid Dynamics, a practical approach, Elsevier BH, Amsterdam, Boston, 2008.

[8] T. Cebeci, P. Bradshaw, Physical and Computational Aspects of Convective Heat Transfer, Springer-Verlag, New York-Berlin-Heidelberg-Tokyo, 1984.

[9] A. Thom, C.J. Apelt, Field Computations in Engineering and Physics, D. Van Nostrand Company, Ltd, London, 1961. 
